\title{
Antimicrobial potential of 3-hydroxy-2-methylene-3-phenylpropionic acid derivatives
}

SONI A. SINGH

SUJATA V. BHAT*

Advanced Research Laboratory in Synthetic and Natural Product Chemistry

V. G. Vaze College

Mumbai University, Mulund (E)

Mumbai-4000081, India

\begin{abstract}
Twenty Baylis-Hillman adducts were synthesized from different aromatic aldehydes and activated vinyl derivatives. The adducts, which are differently substituted 3-hydroxy-2-methylene-3-phenylpropionic acid derivatives, were screened for their antimicrobial activity in vitro by the serial dilution method. Many of these molecules displayed potent antibacterial and antifungal activities. The ease of synthesis from low-cost starting materials along with potent antimicrobial activity of these molecules provide the lead for further improvement of activity and reflect the possibility of therapeutic use.
\end{abstract}

Keywords: 3-hydroxy-2-methylene-3-phenylpropionic acid derivatives, antibacterial activity, antifungal activity, Baylis-Hillman reaction

Microorganisms, particularly bacteria, are becoming resistant to more and more antimicrobial agents. Bacteria found in hospitals appear to be especially resistant and are causing increasing difficulties to patients. Bacterial resistance is currently combated by the discovery of new drugs.

The Baylis-Hillman reaction is an important three-component reaction involving economical construction of a carbon-carbon bond between the $\alpha$-position of an activated-vinyl molecule and an electrophile under the catalytic effect of a tertiary amine (most commonly 1,4-diazabicylo[2.2.2]octane, DABCO) and producing an interesting class of synthetically useful densely functionalized molecules $(1,2)$. Baylis-Hillman adducts, containing chemo-specific functional groups in close proximity, have been extensively used in a number of transformation methodologies (3-9). Recently, several Baylis-Hillman adducts have been evaluated for molluscicidal activity against Biomphalaria glabrata (Say) snails, the intermediate hosts of Schistosomiasis. Among these compounds, 3-hydroxy-2-methylene-3-(4'-nitrophenyl)propanonitrile (compound 4, Table I) had the highest activity, with $I C_{50}=6.64 \mu \mathrm{g} \mathrm{mL}^{-1}(10)$. The 2-phenyl-5-amino-uracil derivative with the Baylis-Hillman side chain was found to display extremely interesting properties with regard to its apoptosis-inducing ability in HL-60 cells (11). A new class of pyrimidinyl agents was synthesized using the Baylis-Hillman strategy and some of these molecules displayed po-

* Correspondence; e-mail: sujata8b@gmail.com 
tent antimalarial activity against Plasmodium falciparum in vitro (12). Several Baylis-Hillman adducts were evaluated for antimalarial activity against chloroquine resistant $P$. falciparum and compound 4 was found to be the most active one (13). High selective leishmanicidal activity of 3-hydroxy-2-methylene-3-(4'-bromophenyl)-propanonitrile and analogous compounds has been reported as well (14).

Amphenicols (2-amino-3-phenyl-propane-1,3-diol derivatives), a class of antibacterials including chloramphenicol and thiamphenicol, display a wide spectrum of antibacterial activity (15). Therefore, 3-hydroxy-2-methylene-3-phenylpropionic acid derivatives, obtained by the Baylis-Hillman condensation, were evaluated for antimicrobial activity. Though many Baylis-Hillman adducts are known, there is no previous report describing the antimicrobial activity of these molecules. We report herein the antimicrobial activity of 3-hydroxy-2-methylene-3-phenylpropionic acid derivatives.

\section{EXPERIMENTAL}

FT-IR spectra were recorded on FT-IR spectrometer (Spectrum One, Perkin-Elmer, USA). ${ }^{1} \mathrm{H}$ NMR spectra were recorded on 60 and $400 \mathrm{MHz}$ spectrometers (EM $360 \mathrm{~L}$ and Mercury Plus, respectively; Varian, USA) in $\mathrm{CDCl}_{3}$. Chemical shifts were measured as $\delta$ units (ppm) relative to tetramethylsilane and coupling constants are expressed in Hertz. Microanalyses were performed in a Carlo Erba Model 1106 elemental analyzer. Silica gel (100-200 mesh) used for column chromatography was activated by heating at $120^{\circ} \mathrm{C}$ for $4 \mathrm{~h}$. The purity of all compounds was established by a single spot on TLC plates (Merck, Germany). Iodine vapours or anisaldehyde-sulfuric acid reagent were used as the developing agent and the solvent system used for TLC analysis was ethyl acetate and $n$-hexane $(2: 8)$. Solvents were purified by standard procedures. All other chemicals used in the study were of analytical grade.

\section{Synthesis of 3-hydroxy-2-methylene-3-phenylpropionic acid derivatives 1-20 through} Baylis-Hillman reaction $(10,13)$. General procedure

A mixture of aromatic aldehyde $(0.6 \mathrm{mmol})$, activated alkene $(2.4 \mathrm{mmol})$ and DABCO $(0.06 \mathrm{mmol})$ was stirred at room temperature for the period mentioned in Table I. The reaction mixture was diluted with ether $(20 \mathrm{~mL})$, washed with $5 \mathrm{~mL}$ of $2 \mathrm{~mol} \mathrm{~L}^{-1} \mathrm{HCl}$ and water. The organic layer was dried (anhydrous $\mathrm{Na}_{2} \mathrm{SO}_{4}$ ). Solvent was evaporated and the residue was purified by column chromatography on silica gel. Corresponding Baylis-Hillman adducts 1-20 were obtained in varying yields (Table I).

\section{Antimicrobial activity}

Minimum inhibitory concentration (MIC). - Broth dilution method was used to determine the MIC of test compounds. Stock solutions of compounds 1-20 were prepared by dissolving $1 \mathrm{mg}$ in $1 \mathrm{~mL}$ of DMSO and diluted to the desired concentration. Fresh cultures of bacteria (Staphylococcus aureus, ATCC 9144; Corynebacterium diphtheriae, ATCC 27010; Klebsiella pneumoniae, ATCC 10031; Escherichia coli, ATCC 10536) and fungi (Aspergillus niger, ATCC 16404 and Candida albicans, ATCC 10231) were prepared. The antibacterial activity 
Table I. 3-Hydroxy-2-methylene-3-phenylpropionic acid derivatives<smiles>[R]C(=C)C(O)c1cc([R3])c([R])cc1[R]</smiles>

\begin{tabular}{ccccccc}
\hline Compd. & $\mathrm{R}_{1}$ & $\mathrm{R}_{2}$ & $\mathrm{R}_{3}$ & $\mathrm{R}_{4}$ & $\begin{array}{c}\text { Time } \\
\text { (days) }\end{array}$ & $\begin{array}{c}\text { Yield } \\
(\%)\end{array}$ \\
\hline $\mathbf{1}$ & $\mathrm{H}$ & $\mathrm{H}$ & $\mathrm{H}$ & $\mathrm{COOMe}$ & 2.00 & 50 \\
$\mathbf{2}$ & $\mathrm{H}$ & $\mathrm{H}$ & $\mathrm{H}$ & $\mathrm{CN}$ & 2.00 & 67 \\
$\mathbf{3}$ & $\mathrm{H}$ & $\mathrm{NO}_{2}$ & $\mathrm{H}$ & $\mathrm{COOMe}$ & 2.00 & 48 \\
$\mathbf{4}$ & $\mathrm{H}$ & $\mathrm{NO}_{2}$ & $\mathrm{H}$ & $\mathrm{CN}$ & 0.08 & 53 \\
$\mathbf{5}$ & $\mathrm{NO}_{2}$ & $\mathrm{H}$ & $\mathrm{H}$ & $\mathrm{COOMe}$ & 3.00 & 58 \\
$\mathbf{6}$ & $\mathrm{NO}_{2}$ & $\mathrm{H}$ & $\mathrm{H}$ & $\mathrm{CN}$ & 3.00 & 62 \\
$\mathbf{7}$ & $\mathrm{H}$ & $\mathrm{Cl}$ & $\mathrm{H}$ & $\mathrm{COOEt}$ & 2.00 & 62 \\
$\mathbf{8}$ & $\mathrm{H}$ & $\mathrm{Cl}$ & $\mathrm{H}$ & $\mathrm{CN}$ & 2.00 & 72 \\
$\mathbf{9}$ & $\mathrm{OMe}$ & $\mathrm{H}$ & $\mathrm{H}$ & $\mathrm{CN}$ & 3.00 & 69 \\
$\mathbf{1 0}$ & $\mathrm{OMe}$ & $\mathrm{H}$ & $\mathrm{H}$ & $\mathrm{COOEt}$ & 3.00 & 65 \\
$\mathbf{1 1}$ & $\mathrm{H}$ & $\mathrm{F}$ & $\mathrm{H}$ & $\mathrm{CN}$ & 2.50 & 66 \\
$\mathbf{1 2}$ & $\mathrm{H}$ & $\mathrm{F}$ & $\mathrm{H}$ & $\mathrm{COOEt}$ & 2.50 & 65 \\
$\mathbf{1 3}$ & $\mathrm{Cl}$ & $\mathrm{H}$ & $\mathrm{H}$ & $\mathrm{CN}$ & 2.00 & 69 \\
$\mathbf{1 4}$ & $\mathrm{Cl}$ & $\mathrm{H}$ & $\mathrm{H}$ & $\mathrm{COOEt}$ & 3.00 & 68 \\
$\mathbf{1 5}$ & $\mathrm{Cl}$ & $\mathrm{Cl}$ & $\mathrm{H}$ & $\mathrm{COOEt}$ & 3.00 & 64 \\
$\mathbf{1 6}$ & $\mathrm{Cl}$ & $\mathrm{Cl}$ & $\mathrm{H}$ & $\mathrm{CN}$ & 3.00 & 64 \\
$\mathbf{1 7}$ & $\mathrm{H}$ & $\mathrm{H}$ & $\mathrm{NO}$ & $\mathrm{COOEt}$ & 3.00 & 68 \\
$\mathbf{1 8}$ & $\mathrm{H}$ & $\mathrm{H}$ & $\mathrm{NO}_{2}$ & $\mathrm{CN}$ & 4.00 & 68 \\
$\mathbf{1 9}$ & $\mathrm{H}$ & $\mathrm{NO}$ & $\mathrm{H}$ & $\mathrm{COOEt}$ & 3.00 & 59 \\
$\mathbf{2 0}$ & $\mathrm{H}$ & $\mathrm{Cl}$ & $\mathrm{H}$ & $\mathrm{COEt}$ & 3.00 & 54 \\
\hline
\end{tabular}

of each test compound was examined as follows: in the test tube containing Müller-Hinton broth (Merck, Germany), a bacterial concentration of $5 \times 10^{5}$ colony forming units $\mathrm{mL}^{-1}$ was tested with 5-30 $\mu \mathrm{g} \mathrm{mL}^{-1}$ (serial dilution) of test compounds. Moreover, a tube of the Müller-Hinton broth containing the same bacterial concentration, but without test compound, was used as growth control and another tube of uninoculated Müller-Hinton broth containing the same concentration of test compound was used as negative growth. Also, a tube of Müller-Hinton broth containing the same bacterial concentration and DMSO, without test compound, was prepared as control to rule out antibacterial effect of DMSO. Test tubes and three mentioned control tubes were incubated at $37^{\circ} \mathrm{C}$ for $24 \mathrm{~h}$. After incubation, antibacterial activity of the test compound in the test tube was detected by lack of turbidity (matching the negative growth control), which indicated inhibition of bacterial growth. The turbidity was measured at $530 \mathrm{~nm}$. 


\section{RESULTS AND DISCUSSION}

In the present work, various substituted benzaldehydes were condensed with activated vinyl derivatives in the presence of a catalytic amount of DABCO according to literature procedures $(10,13)$ (Scheme 1$)$. Various activated vinyl derivatives included ethyl and methyl acrylates, acrylonitrile and ethyl-vinyl-ketone (Scheme 1). The reaction was carried out at room temperature without solvent. The reaction time ranged from 2 to 4 days and 3-hydroxy-2-methylene-3-phenyl-propionic acid derivatives 1-20 were obtained in moderate to good yields (48-72\%, Table I).

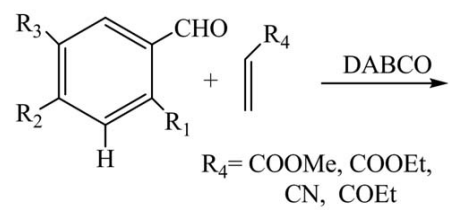<smiles>[R]C(=C)C(O)c1cc([R])c([R])cc1[Y]</smiles>

Scheme 1.

The Baylis-Hillman adducts were characterized using IR, ${ }^{1} \mathrm{H}$ NMR spectra and micro analyses. IR spectra of compounds showed peaks at $\sim 3450$ and $1620 \mathrm{~cm}^{-1}$ (hydroxy and vinyl group, respectively). In the ${ }^{1} \mathrm{H} \mathrm{NMR}$, vinyl protons and allylic proton singlets appeared at $\delta 5.9-6.2$ and $\sim 5.3 \mathrm{ppm}$, respectively. In addition, ${ }^{1} \mathrm{H}$ NMR of all molecules displayed characteristic peaks due to aromatic protons. Microanalyses of synthesized compounds were in agreement with their molecular formula with $\pm 0.3 \%$ (Table II).

\section{Antibacterial activity and structure activity relationship}

Antibacterial activity of the Baylis-Hillman adducts, namely 3-hydroxy-2-methylene-3-phenylpropionic acid derivatives 1-20, was evaluated by the agar serial dilution method (16) against the bacteria and fungi mentioned above. Minimum inhibitory concentrations (MIC, $\mu \mathrm{g} \mathrm{mL} \mathrm{m}^{-1}$ ) of compounds for antibacterial and antifungal activities are given in Table III.

Many of the test compounds displayed good antimicrobial activity; compounds 3-8 and 11-19, showed slighty higher to comparable antimicrobial activity to standard drugs. Compounds 3-6, 15, 17 and 18 showed antimicrobial activity with MIC 14-16 $\mathrm{gg} \mathrm{mL}^{-1}$ vs. chloramphenicol (MIC 21 and $19 \mu \mathrm{g} \mathrm{mL}^{-1}$, respectively) against S. aureus and C. diphtheriae. These active compounds contained 2-, 3- or 4-nitro- (3-6, 17 and 18) or 2,4-dichloro (15) substituents in the phenyl ring. Compounds 15 and 20 displayed antibacterial activity with MIC 15-16 $\mu \mathrm{g} \mathrm{mL}-1$ vs. standard chloramphenicol (MIC $20 \mu \mathrm{g} \mathrm{mL}^{-1}$ ) against K. pneumoniae. Both of these compounds contained 4-chloro-substituent in the phenyl ring. Compounds 7, 8, 12-14 and 17 containing 4-chloro- $(7,8)$, 4-fluoro- $(12), 2$-chloro- $(13,14)$ or 3-nitro(17) in the phenyl ring were potent against E. coli with MIC $15-16 \mu \mathrm{gL}^{-1}$ compared to chloramphenicol (MIC $\left.19 \mathrm{\mu g} \mathrm{mL}^{-1}\right)$. Against $A$. niger compounds 5, 6 (2-nitro-), 7 (4-chloro-), 11 (4-fluoro) and 16 (2,4-dichloro-) with MIC $11 \mu \mathrm{g} \mathrm{mL} \mathrm{mL}^{-1}$ displayed comparable antifungal activity to that of nystatin (MIC $13 \mu \mathrm{g} \mathrm{mL}{ }^{-1}$ ) and against $C$. albicans. Compounds 
S. A. Singh and S. V. Bhat: Antimicrobial potential of 3-hydroxy-2-methylene-3-phenylpropionic acid derivatives, Acta Pharm. 61 (2011) 447-455.

Table II. Spectral and microanalytical data of synthesized compounds

\begin{tabular}{|c|c|c|c|c|c|c|}
\hline \multirow[t]{2}{*}{ Compd. } & \multirow[t]{2}{*}{$\begin{array}{c}\mathrm{IR} \\
\left(v_{\max }, \mathrm{cm}^{-1}\right)\end{array}$} & \multirow[t]{2}{*}{ 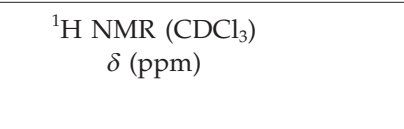 } & \multirow[t]{2}{*}{$\begin{array}{l}\text { Molecular formula } \\
\qquad\left(M_{\mathrm{r}}\right)\end{array}$} & \multicolumn{3}{|c|}{$\begin{array}{l}\text { Elemental analysis } \\
\text { calcd. / found (\%) }\end{array}$} \\
\hline & & & & $\mathrm{C}$ & $\mathrm{H}$ & $\bar{N}$ \\
\hline 1 & $\begin{array}{l}3450,2960 \\
1730,1620 \\
1440,1430\end{array}$ & $\begin{array}{l}7.50-7.20(\mathrm{~m}, 5 \mathrm{H}, \mathrm{Ar}-\mathrm{H}) \\
6.31,5.80\left(\mathrm{~s}, 1 \mathrm{H} \text { each, }-\mathrm{C}=\mathrm{CH}_{2}\right) \\
5.08(\mathrm{~s}, 1 \mathrm{H}, \text { allylic } \mathrm{H}) \\
3.70(\mathrm{~s}, 3 \mathrm{H}, \mathrm{OMe}) \\
3.20 \text { (bs, } 1 \mathrm{H},-\mathrm{OH})\end{array}$ & $\begin{array}{l}\mathrm{C}_{11} \mathrm{H}_{12} \mathrm{O}_{3} \\
(192.21)\end{array}$ & $\begin{array}{l}68.74 \\
68.45\end{array}$ & $\begin{array}{l}6.29 \\
6.48\end{array}$ & - \\
\hline 2 & $\begin{array}{l}3460,2854 \\
2224,1585 \\
965\end{array}$ & $\begin{array}{l}\text { 7.50-7.20 (m, 5H, Ar-H), } \\
6.10\left(\mathrm{~s}, 2 \mathrm{H}-\mathrm{C}=\mathrm{CH}_{2}\right) \\
5.83(\mathrm{~s}, 1 \mathrm{H}, \text { allylic } \mathrm{H}) \\
3.20 \text { (bs, } 1 \mathrm{H},-\mathrm{OH})\end{array}$ & $\begin{array}{c}\mathrm{C}_{10} \mathrm{H}_{9} \mathrm{NO} \\
(159.18)\end{array}$ & $\begin{array}{l}75.45 \\
75.66\end{array}$ & $\begin{array}{l}5.70 \\
5.41\end{array}$ & $\begin{array}{l}8.80 \\
8.68\end{array}$ \\
\hline 3 & $\begin{array}{l}3460,2960 \\
1730,1630 \\
1610,1510\end{array}$ & $\begin{array}{l}7.95(\mathrm{~d}, J=10 \mathrm{~Hz}, 2 \mathrm{H}, \mathrm{Ar}-\mathrm{H}) \\
7.55(\mathrm{~d}, J=10 \mathrm{~Hz}, 2 \mathrm{H}, \mathrm{Ar}-\mathrm{H}) \\
6.38,5.90\left(\mathrm{~s}, 1 \mathrm{H} \text { each, }-\mathrm{C}=\mathrm{CH}_{2}\right) \\
5.62(\mathrm{~s}, 1 \mathrm{H}, \text { allylic H}) \\
3.72(\mathrm{~s}, 3 \mathrm{H}, \mathrm{OMe}) \\
3.40 \text { (bs, } 1 \mathrm{H},-\mathrm{OH})\end{array}$ & $\begin{array}{l}\mathrm{C}_{11} \mathrm{H}_{11} \mathrm{NO}_{5} \\
\quad(237.21)\end{array}$ & $\begin{array}{l}55.70 \\
55.97\end{array}$ & $\begin{array}{l}4.67 \\
4.87\end{array}$ & $\begin{array}{l}5.90 \\
5.72\end{array}$ \\
\hline 4 & $\begin{array}{l}3447,2959, \\
2227,1610 \\
1530,952\end{array}$ & $\begin{array}{l}7.95(\mathrm{~d}, J=9 \mathrm{~Hz}, 2 \mathrm{H}, \mathrm{Ar}-\mathrm{H}) \\
7.60(\mathrm{~d}, J=9 \mathrm{~Hz}, 2 \mathrm{H}, \mathrm{Ar}-\mathrm{H}) \\
6.15\left(\mathrm{~s}, 2 \mathrm{H},-\mathrm{C}=\mathrm{CH}_{2}\right) \\
5.48(\mathrm{bs}, 1 \mathrm{H}, \text { allylic } \mathrm{H}) \\
3.08(\mathrm{~s}, 1 \mathrm{H},-\mathrm{OH})\end{array}$ & $\begin{array}{l}\mathrm{C}_{10} \mathrm{H}_{8} \mathrm{~N}_{2} \mathrm{O}_{3} \\
\quad(204.18)\end{array}$ & $\begin{array}{l}58.82 \\
58.68\end{array}$ & $\begin{array}{l}3.95 \\
3.74\end{array}$ & $\begin{array}{l}13.72 \\
13.43\end{array}$ \\
\hline 5 & $\begin{array}{l}3440,2954, \\
1730,1630 \\
1610,1510\end{array}$ & $\begin{array}{l}\text { 7.45-7.75 (m, 4H, Ar-H), } \\
6.60,6.20\left(\mathrm{~s}, 1 \mathrm{H} \text { each, }-\mathrm{C}=\mathrm{CH}_{2}\right) \\
5.82(\mathrm{~s}, 1 \mathrm{H}, \text { allylic } \mathrm{H}) \\
5.20(\mathrm{~s}, 3 \mathrm{H}, \mathrm{OMe}) \\
2.40(\mathrm{bs}, 1 \mathrm{H},-\mathrm{OH})\end{array}$ & $\begin{array}{l}\mathrm{C}_{11} \mathrm{H}_{11} \mathrm{NO}_{5} \\
\quad(237.21)\end{array}$ & $\begin{array}{l}55.70 \\
55.57\end{array}$ & $\begin{array}{l}4.57 \\
4.28\end{array}$ & $\begin{array}{l}5.90 \\
5.65\end{array}$ \\
\hline 6 & $\begin{array}{l}3440,2940 \\
2227,1610 \\
1530\end{array}$ & $\begin{array}{l}7.90-7.2(\mathrm{~m}, 4 \mathrm{H}, \mathrm{Ar}-\mathrm{H}) \\
6.35\left(\mathrm{~s}, 2 \mathrm{H},-\mathrm{C}=\mathrm{CH}_{2}\right) \\
5.58(\mathrm{bs}, 1 \mathrm{H}, \text { allylic } \mathrm{H}) \\
3.40(\mathrm{~s}, 1 \mathrm{H},-\mathrm{OH})\end{array}$ & $\begin{array}{l}\mathrm{C}_{10} \mathrm{H}_{8} \mathrm{~N}_{2} \mathrm{O}_{3} \\
\quad(204.18)\end{array}$ & $\begin{array}{l}58.82 \\
58.64\end{array}$ & $\begin{array}{l}3.95 \\
3.68\end{array}$ & $\begin{array}{l}13.72 \\
13.45\end{array}$ \\
\hline 7 & $\begin{array}{l}3500,2960 \\
1736,1630 \\
1610,1510\end{array}$ & $\begin{array}{l}7.85(\mathrm{~d}, J=9 \mathrm{~Hz}, 2 \mathrm{H}, \mathrm{Ar}-\mathrm{H}), \\
7.35(\mathrm{~d}, J=9 \mathrm{~Hz}, 2 \mathrm{H}, \mathrm{Ar}-\mathrm{H}) \\
6.38,5.95\left(\mathrm{~s}, 1 \mathrm{H}, \text { each, }-\mathrm{C}=\mathrm{CH}_{2}\right) \\
5.82(\mathrm{~s}, 1 \mathrm{H}, \text { allylic } \mathrm{H}), \\
4.20\left(\mathrm{q}, J=7 \mathrm{~Hz}, 2 \mathrm{H},-\mathrm{OCH}_{2}\right), \\
3.20(\mathrm{bs}, 1 \mathrm{H},-\mathrm{OH}), \\
1.30(\mathrm{t}, J=7 \mathrm{~Hz}, 3 \mathrm{H}, \mathrm{Me})\end{array}$ & $\begin{array}{l}\mathrm{C}_{1} \mathrm{C}_{12} \mathrm{H}_{13} \mathrm{ClO}_{3} \\
(240.68)\end{array}$ & $\begin{array}{l}59.88 \\
59.59\end{array}$ & $\begin{array}{l}5.44 \\
5.65\end{array}$ & $\begin{array}{l}14.73 \\
14.83\end{array}$ \\
\hline 8 & $\begin{array}{l}3440,2969 \\
2246,1610 \\
1530\end{array}$ & $\begin{array}{l}7.95(\mathrm{~d}, J=9 \mathrm{~Hz}, 2 \mathrm{H}, \mathrm{Ar}-\mathrm{H}) \\
7.60(\mathrm{~d}, J=9 \mathrm{~Hz}, 2 \mathrm{H}, \mathrm{Ar}-\mathrm{H}) \\
6.38,5.95\left(\mathrm{~s}, 1 \mathrm{H}, \text { each, }-\mathrm{C}=\mathrm{CH}_{2}\right) \\
5.58(\mathrm{bs}, 1 \mathrm{H}, \text { allylic H}) \\
3.04(\mathrm{~s}, 1 \mathrm{H},-\mathrm{OH})\end{array}$ & $\begin{array}{l}\mathrm{C}_{10} \mathrm{H}_{8} \mathrm{ClNO} \\
(193.63)\end{array}$ & $\begin{array}{l}62.03 \\
62.23\end{array}$ & $\begin{array}{l}4.16 \\
4.38\end{array}$ & $\begin{array}{l}7.23 \\
7.52\end{array}$ \\
\hline 9 & $\begin{array}{l}3440,2940 \\
2247,1667 \\
1610,1530\end{array}$ & $\begin{array}{l}\text { 7.95-7.60 (m, 4H, Ar-H), } \\
6.18,5.75\left(\mathrm{~s}, 1 \mathrm{H} \text { each, }-\mathrm{C}=\mathrm{CH}_{2}\right) \\
5.48 \text { (bs, } 1 \mathrm{H}, \text { allylic } \mathrm{H}) \\
3.40(\mathrm{~s}, 1 \mathrm{H},-\mathrm{OH}) \\
3.42(\mathrm{~s}, 3 \mathrm{H}, \mathrm{OMe})\end{array}$ & $\begin{array}{l}\mathrm{C}_{11} \mathrm{H}_{11} \mathrm{NO}_{2} \\
\quad(189.21)\end{array}$ & $\begin{array}{l}69.83 \\
69.54\end{array}$ & $\begin{array}{l}3.86 \\
3.66\end{array}$ & $\begin{array}{l}7.40 \\
7.57\end{array}$ \\
\hline
\end{tabular}




\begin{tabular}{|c|c|c|c|c|c|c|}
\hline 10 & $\begin{array}{l}3440,2960 \\
1730,1630 \\
1610,1510\end{array}$ & $\begin{array}{l}7.95-7.55(\mathrm{~m}, 4 \mathrm{H}, \mathrm{Ar}-\mathrm{H}), \\
6.68,6.12\left(\mathrm{~s}, 1 \mathrm{H} \text { each, }-\mathrm{C}=\mathrm{CH}_{2}\right), \\
5.85(\mathrm{~s}, 1 \mathrm{H}, \text { allylic- } \mathrm{H}), \\
4.20\left(\mathrm{q}, J=7 \mathrm{~Hz}, 2 \mathrm{H},-\mathrm{OCH}_{2}-\right) \\
3.62(\mathrm{~s}, 3 \mathrm{H}, \mathrm{OMe}) \\
3.05(\mathrm{bs}, 1 \mathrm{H},-\mathrm{OH}), \\
1.45(\mathrm{t}, J=7 \mathrm{~Hz}, 3 \mathrm{H}, \mathrm{Me})\end{array}$ & $\begin{array}{c}\mathrm{C}_{13} \mathrm{H}_{16} \mathrm{O}_{4} \\
(236.26)\end{array}$ & $\begin{array}{l}66.09 \\
65.87\end{array}$ & $\begin{array}{l}6.83 \\
6.55\end{array}$ & $\begin{array}{l}- \\
-\end{array}$ \\
\hline 11 & $\begin{array}{l}3440,2940, \\
2247,1610, \\
1530\end{array}$ & $\begin{array}{l}7.95(\mathrm{~m}, 2 \mathrm{H}, \mathrm{Ar}-\mathrm{H}) \\
7.30(\mathrm{~d}, J=8.5 \mathrm{~Hz}, 2 \mathrm{H}, \mathrm{Ar}-\mathrm{H}) \\
6.35\left(\mathrm{~d}, 2 \mathrm{H},-\mathrm{C}=\mathrm{CH}_{2}\right) \\
5.58(\mathrm{bs}, 1 \mathrm{H}, \text { allylic } \mathrm{H}) \\
3.04(\mathrm{~s}, 1 \mathrm{H},-\mathrm{OH})\end{array}$ & $\begin{array}{c}\mathrm{C}_{10} \mathrm{H}_{8} \mathrm{FNO} \\
(177.18)\end{array}$ & $\begin{array}{l}67.70 \\
67.54\end{array}$ & $\begin{array}{l}4.55 \\
4.78\end{array}$ & $\begin{array}{l}7.91 \\
7.68\end{array}$ \\
\hline 12 & $\begin{array}{l}3500,2960 \\
1738,1630 \\
1610,1510\end{array}$ & $\begin{array}{l}7.95(\mathrm{~m}, 2 \mathrm{H}, \mathrm{Ar}-\mathrm{H}) \\
7.30(\mathrm{~d}, J=8.5 \mathrm{~Hz}, 2 \mathrm{H}, \mathrm{Ar}-\mathrm{H}) \\
6.60,6.15\left(\mathrm{~s}, 1 \mathrm{H},-\mathrm{C}=\mathrm{CH}_{2}\right) \\
5.85(\mathrm{~s}, 1 \mathrm{H}, \text { allylic } \mathrm{H}) \\
4.42\left(\mathrm{q}, J=7 \mathrm{~Hz}, 2 \mathrm{H},-\mathrm{OCH}_{2}-\right) \\
3.20(\mathrm{bs}, 1 \mathrm{H},-\mathrm{OH}) \\
1.40(\mathrm{t}, J=7 \mathrm{~Hz}, 3 \mathrm{H}, \mathrm{Me})\end{array}$ & $\begin{array}{c}\mathrm{C}_{12} \mathrm{H}_{13} \mathrm{FO}_{3} \\
(224.23)\end{array}$ & $\begin{array}{l}64.28 \\
64.56\end{array}$ & $\begin{array}{l}5.84 \\
5.58\end{array}$ & $\begin{array}{l}- \\
-\end{array}$ \\
\hline 13 & $\begin{array}{l}3440,2940, \\
2228,1610, \\
1530\end{array}$ & $\begin{array}{l}7.70-7.20(\mathrm{~m}, 4 \mathrm{H}, \mathrm{Ar}-\mathrm{H}) \\
5.85\left(\mathrm{~s}, 2 \mathrm{H},-\mathrm{C}=\mathrm{CH}_{2}\right) \\
5.28(\mathrm{~s}, 1 \mathrm{H}, \text { allylic } \mathrm{H}) \\
2.90(\mathrm{~s}, 1 \mathrm{H},-\mathrm{OH})\end{array}$ & $\begin{array}{c}\mathrm{C}_{10} \mathrm{H}_{8} \mathrm{ClNO} \\
(193.63)\end{array}$ & $\begin{array}{l}62.03 \\
62.29\end{array}$ & $\begin{array}{l}4.16 \\
4.42\end{array}$ & $\begin{array}{l}7.23 \\
7.52\end{array}$ \\
\hline 14 & $\begin{array}{l}3500,2960 \\
1736,1648, \\
1617,1530\end{array}$ & $\begin{array}{l}7.95-7.65(\mathrm{~m}, 4 \mathrm{H}, \mathrm{Ar}-\mathrm{H}), \\
6.38,5.90\left(\mathrm{~s}, 1 \mathrm{H} \text { each, }-\mathrm{C}=\mathrm{CH}_{2}\right) \\
5.82(\mathrm{~s}, 1 \mathrm{H}, \text { allylic } \mathrm{H}), \\
3.42\left(\mathrm{q}, J=7 \mathrm{~Hz}, 2 \mathrm{H},-\mathrm{OCH}_{2^{-}}\right) \\
3.04(\mathrm{bs}, 1 \mathrm{H},-\mathrm{OH}) \\
1.60(\mathrm{t}, J=7 \mathrm{~Hz}, 3 \mathrm{H}, \mathrm{Me})\end{array}$ & $\begin{array}{c}\mathrm{C}_{12} \mathrm{H}_{13} \mathrm{ClO}_{3} \\
(240.68)\end{array}$ & $\begin{array}{l}59.88 \\
59.67\end{array}$ & $\begin{array}{l}5.44 \\
5.71\end{array}$ & $\begin{array}{l}- \\
-\end{array}$ \\
\hline 15 & $\begin{array}{l}3500,2960 \\
1740,1630 \\
1610,1510\end{array}$ & $\begin{array}{l}8.85(\mathrm{~s}, 1 \mathrm{H}, \mathrm{Ar}-\mathrm{H}), \\
7.70-7.45(\mathrm{bd}, 2 \mathrm{H}, \mathrm{Ar}-\mathrm{H}), \\
6.38,5.90\left(\mathrm{~s}, 1 \mathrm{H} \text { each, }-\mathrm{C}=\mathrm{CH}_{2}\right), \\
5.82(\mathrm{~s}, 1 \mathrm{H}, \text { allylic } \mathrm{H}), \\
3.42\left(\mathrm{q}, J=7 \mathrm{~Hz}, 2 \mathrm{H},-\mathrm{OCH}_{2}-\right), \\
3.40(\mathrm{bs}, 1 \mathrm{H},-\mathrm{OH}), \\
1.60(\mathrm{t}, J=7 \mathrm{~Hz}, 3 \mathrm{H}, \mathrm{Me})\end{array}$ & $\begin{array}{c}\mathrm{C}_{12} \mathrm{H}_{12} \mathrm{Cl}_{2} \mathrm{O}_{3} \\
(275.13)\end{array}$ & $\begin{array}{l}52.39 \\
52.68\end{array}$ & $\begin{array}{l}4.40 \\
4.69\end{array}$ & $\begin{array}{l}- \\
-\end{array}$ \\
\hline 16 & $\begin{array}{l}3440,2940, \\
2247,1610, \\
1530\end{array}$ & $\begin{array}{l}7.85(\mathrm{~s}, 1 \mathrm{H}, \mathrm{Ar}-\mathrm{H}) \\
7.70-7.45(\mathrm{bd}, 2 \mathrm{H}, \mathrm{Ar}-\mathrm{H}) \\
6.35\left(\mathrm{~s}, 2 \mathrm{H},-\mathrm{C}=\mathrm{CH}_{2}\right) \\
5.08 \text { (bs, 1H, allylic } \mathrm{H}) \\
3.40 \text { (s, } 1 \mathrm{H},-\mathrm{OH})\end{array}$ & $\begin{array}{c}\mathrm{C}_{10} \mathrm{H}_{7} \mathrm{Cl}_{2} \mathrm{NO} \\
(228.07)\end{array}$ & $\begin{array}{l}52.66 \\
52.87\end{array}$ & $\begin{array}{l}3.09 \\
3.36\end{array}$ & $\begin{array}{l}6.14 \\
6.35\end{array}$ \\
\hline 17 & $\begin{array}{l}3500,2960 \\
1730,1630 \\
1610,1510\end{array}$ & $\begin{array}{l}8.45(\mathrm{~s}, 1 \mathrm{H}, \mathrm{Ar}-\mathrm{H}) \\
8.40-7.70(\mathrm{~m}, 3 \mathrm{H}, \mathrm{Ar}-\mathrm{H}), \\
6.48,5.70\left(\mathrm{~s}, 1 \mathrm{H} \text { each, }-\mathrm{C}=\mathrm{CH}_{2}\right) \\
5.42(\mathrm{~s}, 1 \mathrm{H}, \text { allylic } \mathrm{H}), \\
3.72\left(\mathrm{q}, J=7 \mathrm{~Hz}, 2 \mathrm{H},-\mathrm{OCH}_{2}-\right) \\
3.04(\mathrm{bs}, 1 \mathrm{H},-\mathrm{OH}) \\
1.60(\mathrm{t}, J=7 \mathrm{~Hz}, 3 \mathrm{H}, \mathrm{Me})\end{array}$ & $\begin{array}{c}\mathrm{C}_{12} \mathrm{H}_{13} \mathrm{NO}_{5} \\
(251.24)\end{array}$ & $\begin{array}{l}57.37 \\
57.59\end{array}$ & $\begin{array}{l}5.22 \\
5.48\end{array}$ & $\begin{array}{l}5.58 \\
5.81\end{array}$ \\
\hline
\end{tabular}




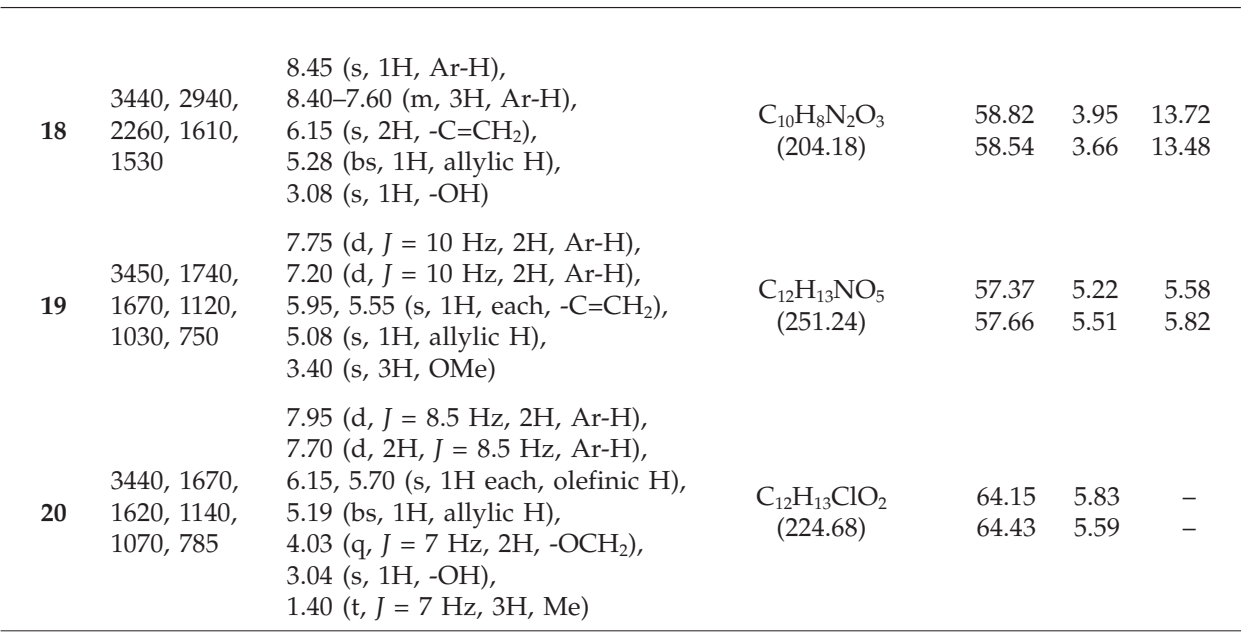

Table III. Minimum inhibitory concentration (MIC) of compounds 1-20a

\begin{tabular}{|c|c|c|c|c|c|c|}
\hline Compd. & S. aureus & C. diphtheriae & K. pneumoniae & E. coli & A. niger & C. albicans \\
\hline 1 & - & 25 & 30 & 22 & - & - \\
\hline 2 & 20 & 20 & 21 & 19 & - & - \\
\hline 3 & 16 & 14 & 18 & 17 & - & 13 \\
\hline 4 & 15 & 15 & 20 & 17 & 12 & 12 \\
\hline 5 & 16 & 14 & 18 & 17 & 11 & 12 \\
\hline 6 & 15 & 15 & 20 & 17 & 11 & 13 \\
\hline 7 & 18 & 16 & 18 & 15 & 11 & 12 \\
\hline 8 & 16 & 19 & 18 & 16 & 12 & 13 \\
\hline 9 & - & - & 25 & 24 & 12 & - \\
\hline 10 & - & - & 22 & 21 & - & 12 \\
\hline 11 & 18 & 18 & 20 & 17 & 11 & 13 \\
\hline 12 & 20 & 19 & 18 & 16 & 14 & 15 \\
\hline 13 & 18 & 18 & 18 & 16 & 14 & 13 \\
\hline 14 & 18 & 18 & 18 & 16 & 14 & 13 \\
\hline 15 & 16 & 15 & 15 & 18 & 13 & 11 \\
\hline 16 & 18 & 17 & 18 & 20 & 11 & 15 \\
\hline 17 & 16 & 14 & 18 & 16 & 13 & 14 \\
\hline 18 & 16 & 14 & 18 & 17 & 14 & 10 \\
\hline 19 & 18 & 18 & 17 & 18 & 12 & 11 \\
\hline 20 & 18 & 18 & 16 & 20 & 13 & 12 \\
\hline Standard ${ }^{\mathrm{b}}$ & 21 & 19 & 20 & 19 & 13 & 15 \\
\hline
\end{tabular}

${ }^{\mathrm{a}} \mu \mathrm{g} \mathrm{mL}^{-1}$

b Chloramphenicol and nystatin were used as reference standards for antibacterial and antifungal tests respectively. 
15 (2,4-dichloro), 18 and 19 (3-nitro-) with MIC 10-11 $\mu \mathrm{g} \mathrm{mL} \mathrm{mL}^{-1}$ showed good antifungal activity compared to nystatin (MIC $15 \mu \mathrm{g} \mathrm{mL} \mathrm{m}^{-1}$ ).

The adducts with 2-methoxy-substituent (9 and 10) did not display any antibacterial activity but showed antifungal activity with MIC $12 \mu \mathrm{g} \mathrm{mL}-1$ against $A$. niger and $C$. albicans, respectively.

The compounds with 4-fluoro-substituent (11 and 12) and with 4-chloro-substituent (7 and 8) displayed similar antibacterial and antifungal activities, whereas adducts 1 and 2 , without any substituent in the phenyl ring, were devoid of antimicrobial activity.

\section{CONCLUSIONS}

Many among differently substituted 3-hydroxy-2-methylene-3-phenylpropionic acid derivatives displayed good antimicrobial activity. Particularly, the 3-hydroxy-2-methylene-3-phenylpropionic acid derivatives with 2-, 3- or 4-nitro- $(3-6,17-19), 2$-chloro- $(13,14)$, 4-chloro $(\mathbf{7}, \mathbf{8}, \mathbf{2 0})$, 4-fluoro- $(\mathbf{1 1}, \mathbf{1 2})$ and 2,4-dichloro- $(\mathbf{1 5}, \mathbf{1 6})$ substituents in the phenyl ring helped in potent antibacterial and/or antifugal activities. The derivatives with 2-methoxy substituent $(9, \mathbf{1 0})$ in the phenyl ring displayed only antifugal activity. The ease of synthesis from low cost starting materials along with potent antimicrobial activity of these molecules provide the lead for further improvement of activity and reflect the possibility of therapeutic use.

Acknowledgements. - The authors are grateful to S. H. Kelkar Co. for financial assistance and Ana Laboratories, India, for their support to carry out antimicrobial activity evaluations. We are also thankful to the Department of Chemistry and Sophisticated Analytical Instrumentation Facility, Indian Institute of Technology Bombay, India, for NMR spectral data.

\section{REFERENCES}

1. A. B. Baylis and M. E. D. Hillman, German Pat. 2155113, May 1972; Chem. Abstr. 77 (1972) 34174q.

2. M. L. Bode and P. T. Kaye, Catalytic enantioselective Baylis-Hillman reactions, correlation between pressure and enantiomeric excess, Tetrahedron Lett. 32 (1991) 5611-5612; DOI: 10.1016/ 0040-4039(91)80098-Q.

3. P. Langer, New strategies for the development of an asymmetric version of the Baylis-Hillman reaction, Angew. Chem. Int. Ed. 39 (2000) 3049-3052; DOI: 10.1002/chin.200047250.

4. S. E. Drewes and G. H. P. Roos, Synthetic potential of the tertiary-amine-catalyzed reaction of activated vinyl carbanions with aldehydes, Tetrahedron 44 (1988) 4653-4670; DOI: 10.1016/S00404020(01)86168-8.

5. M. Shi, C-Q. Li and J-K. Jiang, Different reaction patterns in the Baylis-Hillman reaction of aryl aldehydes with phenyl vinyl ketone, phenyl acrylate and phenyl thioacrylate, Molecules 7 (2002) 721-733; DOI: 10.3390/71000721.

6. K. Y. Lee, S. Gowrisankar and J. N. Kim, Baylis-Hillman reaction and chemical transformations of Baylis-Hillman adducts, Bull. Korean Chem. Soc. 26 (2005) 1481-1490; DOI: 10.1002/chin.200608234.

7. D. Basavaiah, A. J. Rao and T. Satyanarayana, Recent advances in the Baylis-Hillman reaction and applications, Chem. Rev. 103 (2003) 811-820; DOI: 10.1021/cr010043d. 
8. V. Singh and S. A. Batra, Advances in the Baylis-Hillman reaction-assisted synthesis of cyclic frameworks, Tetrahedron 64 (2008) 4511-4574; DOI: 10.1016/j.tet.2008.02.087.

9. D. Roy and R. B. Sunoj, Water-catalysis in Morita-Baylis-Hillman reaction: a mechanistic perspective, Chem. A Europ. J. 14 (2008) 10530-10534; DOI: 10.1002/chem.200801822.

10. M. L. A. A. Vasconcellos, T. M. S. Silva, C. A. Camara, R. M. Martins, K. M. Lacerda, H. M. Lopes, V. L. P. Pereira, R. O. M. A. De Souza and L. T. C. Crespo, Baylis-Hillman adducts with molluscicidal activity against Biomphalaria glabrata, Pest Manag. Sci. 62 (2006) 288-291; DOI: $10.1002 /$ ps. 1153 .

11. D. S. Hamilton, Z. Ding, B. Ganem and D. J. Creighton, Glutathionyl transferase catalyzed addition of glutathione to COMC: a new hypothesis for antitumor activity, Org. Lett. 4 (2002) 1209-1212; DOI: $10.1021 /$ ol025650h.

12. A. G. Gonzalez, M. H. Silva, J. I. Padron, F. Leon, E. Reyes, M. Alvarez-Mon, J. P. Pivel, J. Quintana, F. Estevez and J. Bermejo, Synthesis and antiproliferative activity of a new compound containing an $\alpha$-methylene- $\gamma$-lactone group, J. Med. Chem. 45 (2002) 2358-2359; DOI: 10.1021/ jm025518n.

13. M. K. Kundu, N. Sundar, S. K. Kumar, S. V. Bhat, S. Biswas and N. Valecha, Antimalarial activity of 3-hydroxyalkyl-2-methylene-propionic acid derivatives, Bioorg. Med. Chem. Lett. 9 (1999) 731-736; DOI: 10.1002/chin.199926102.

14. R.O.M.A. de Souza, V. L. P. Pereira, M. F. Mazitano, C. A. B. Falco, B. Rossi-Bergmann, E. B. A. Filho and M. L. A. A. Vase, High selective leishmanicidal activity of 3-hydroxy-2-methylene3-(4-bromophenyl)-propanenitrile and analogous compounds, Eur. J. Med. Chem. 42 (2007) 99-102; DOI: 10.1016/j.ejmech.2006.07.013.

15. I. Shalit and M. I. Marks, Review of pharmacology and clinical efficacy of chloramphenicol, Drugs 28 (1984) 281-291; DOI: 10.2165/00003495-198428040-00001.

16. K. J. Ryan and C. G. Ray (Eds.), Sherris Medical Microbiology, 4th ed., McGraw Hill, New York 2004.

\section{$S A \check{Z} E T A K$}

\section{Antimikrobno djelovanje derivata 3-hidroksi-2-metilen-3-fenilpropionske kiseline}

SONI A. SINGH i SUJATA V. BHAT

Iz različitih aromatskih aldehida i aktiviranih vinil derivata sintetizirano je dvadeset Baylis-Hillmanovih adukata, različito supstituiranih derivata 3-hidroksi-2-metilen-3-fenilpropionske kiseline. Ispitano je njihovo antimikrobno djelovanje in vitro metodom serijskih razrjeđenja. Većina ispitivanih spojeva pokazala je snažno antibakterijsko i antifungalno djelovanje. Jednostavni sintetski postupak iz jeftinih sirovina i snažno antimikrobno djelovanje pružaju vodeće spojeve za daljnju modifikaciju, koji mogu dovesti do ljekovitih tvari pogodnih za terapijsku primjenu.

Ključne riječi: derivati 3-hidroksi-2-metilen-3-fenilpropionske kiseline, antibakterijsko djelovanje, antifungalno djelovanje, Baylis-Hillmanova reakcija

Advanced Research Laboratory in Synthetic and Natural Product Chemistry, V. G. Vaze College Mumbai University, Mithagar Road, Mulund (E), Mumbai-4000081, India 